\title{
Sleep and Development
}

\author{
Royi Gilad1 (ㅇ), Colin Shapiro1,2 \\ ${ }^{1}$ Youthdale Child and Adolescent Sleep Center, Toronto, Ontario, Canada \\ ${ }^{2}$ Department of Psychiatry and Ophthalmology, University of Toronto, Toronto, Ontario, Canada \\ Email: royi@youthdalesleepcenter.ca
}

How to cite this paper: Gilad, R. and Shapiro, C. (2020) Sleep and Development. Health, 12, 653-670.

https://doi.org/10.4236/health.2020.126049

Received: April 29, 2019

Accepted: June 26, 2020

Published: June 29, 2020

Copyright (c) 2020 by author(s) and Scientific Research Publishing Inc. This work is licensed under the Creative Commons Attribution International License (CC BY 4.0).

http://creativecommons.org/licenses/by/4.0/

\begin{abstract}
The early years of life are characterized by significant developmental processes of growth and differentiation in all physical systems and especially the central nervous system. Sleep plays a crucial role in cerebral developmental processes and the accumulation of new abilities and skills. We review the current research evidence regarding the relationship between sleep (and its various components) and cerebral and physical developmental processes. Although sleep is an everyday physiological necessity, the ability to recognize it as a critical component of development may bring a different view towards sleep by parents, educators, and caregivers. This change might lead to a shift in individual and social attitudes aimed at enabling the necessary time and conditions for adequate sleep in children and adolescents to become the norm. These sets of values will then hopefully extend into adulthood, in which sleep also plays a crucial role in both physical and mental wellbeing.
\end{abstract}

\section{Keywords}

Sleep, Development, Growth

\section{Introduction}

The first two decades of life are characterized by tremendous, complex biological processes, which induce and control all the changes, which are necessary for highly differentiated organisms, with tissues, organs and organ systems which evolve from a single cell. These processes involve growth and development. Growth is an increase in at least one measurable dimension, e.g. by an increase of cell number, cell size or increase of mass of non-cellular substances. Development is a progression from a simpler or lower stage to a more advanced or mature form or stage. Development comprises differentiation (cells aggregate into tissues, organs and organ systems) and maturation, a process leading to the attainment of full functional capacity [1]. One of these organ systems is the Cen- 
tral Nervous System (CNS) where among other processes, internal and external stimuli are integrated, responses of the body are prepared and where communication with the second great control system of the human body, the endocrine system, takes place. One of the complex behaviors, which are regulated by the brain, is the sleep-wake cycle (and within sleep, the NREM-REM sleep cycle) [1].

\section{The Relation between Growth and Sleep}

The phase of growth and development is divided into a prenatal and a postnatal phase. The most dramatic changes occur prenatally. The prenatal phase is subdivided into an embryonic (week 3 to 8 after conception) and a fetal (week 9 until birth) period. The Nervous system, including the brain, starts to develop very early during embryogenesis and continuous during fetogenesis. During prenatal life embryogenesis is vulnerable to major morphological damages while fetogenesis is vulnerable to the development of functional and minor morphological abnormalities.

During normal prenatal development brain volume doubles during the third trimester of gestation and the volume of gray cortical matter shows a fourfold increase [2]. From the very beginning of life differences between males and females start to emerge. Head circumference at birth, which is a proxy of prenatal brain growth and development is $0.6 \mathrm{~cm}$ larger in boys (median: $34.5 \mathrm{~cm}$ ) than in girls (median: $33.9 \mathrm{~cm}$ ) [3]. The increase in head circumference as a proxy of brain growth is most marked for the first year of life. Of the typical $16 \mathrm{~cm}$ postnatal increase in head circumference during the first five years of life, more than $50 \%$ is completed by six months of age and more than $80 \%$ [3] at the age of two years. It seems apparent that in early life the growth of neural components is far ahead of the general growth of the body.

According to MRI data, brain weight shows the fastest growth in the first three years of life. At the age of five years $90 \%$ of adult brain weight is attained and between 10 and 12 years of age adult values are reached [4].

The microarchitecture of sleep is composed of 2 distinct sleep stages, Non-REM sleep and REM (Rapid Eye Movement) Sleep. Non-REM sleep is differentiated by three sleep stage (N1, N2, N3) [5]. The first stage (N1), is the typical transition from wakefulness to sleep. It is characterized by low amplitude mixed EEG frequencies in the theta range ( 4 to $7 \mathrm{~Hz}$ ) for at least 50 percent of the epoch. Eye movements are typically slow and rolling. Stage N1 is the lightest stage of sleep; patients awakened from it usually do not perceive that they were actually asleep. Stage N1 sleep typically accounts for 5 to 10 percent or less of the total sleep time in young adults. The second stage (N2), generally comprises the largest percentage of total sleep time in a normal middle-aged adult, typically 45 to 55 percent of the night. It is characterized by theta EEG frequency. There are two distinct features of NREM sleep that appear for the first time on the EEG during stage N2: sleep spindles and K-complexes. Sleep spindles are generally short (though at least 0.5 seconds) and have an EEG frequency of 11 to $16 \mathrm{~Hz}$ (most commonly 
12 to $14 \mathrm{~Hz}$ ). They occur most prominently in the central (vertex) EEG leads. $\mathrm{K}$-complexes are well-delineated, negative, sharp waves immediately followed by a positive component that stand out from the background EEG and have a total duration of $\geq 0.5$ seconds. A K-complex usually has maximal amplitude in the frontal regions of the EEG. The third stage of non-REM sleep (N3) sleep is frequently referred to as "deep sleep" or "Slow Wave Sleep" (SWS). It is characterized by low frequency ( 0.5 to $2 \mathrm{~Hz}$ ), high amplitude delta EEG waves with an amplitude $>75$ microvolts, comprising at least 20 percent of a given sleep epoch. Stage N3 sleep typically accounts for 10 to 20 percent of the total sleep time in young to middle age adults and decreases with age. Stage N3 tends to occur more in the first half of the night and particularly at the beginning of the night, since slow wave activity during sleep represents the homeostatic drive to sleep, which is maximal after the waking period [5]. It is often more difficult to arouse sleepers during stage N3 sleep compared with stages N1 and N2, and stage N3 is a typical time for NREM-parasomnias to occur.

REM sleep (stage R), is characterized by three primary features that require EEG, electro-oculography (EOG) and electromyography (EMG) to capture. EEG demonstrates a low voltage, mixed EEG pattern. Sawtooth waves are a common finding during REM sleep; these $2-6 \mathrm{~Hz}$ wave patterns are sharply contoured and occur in brief bursts. Rapid eye movements are the defining feature of the stage. These are defined on EOG by conjugate, irregular, sharply-peaked eye movements with an initial phase less than 500 milliseconds. EMG demonstrates atonia, indicating inactivity of all voluntary muscles (except the extraocular muscles and the diaphragm). Atonia is the result of direct inhibition of alpha motor neurons [6].

REM sleep is sub-segmented into phasic REM sleep and tonic REM sleep. Phasic REM sleep is the portion of REM sleep during which there are bursts of rapid eye movements, which may be associated with brief bursts of EMG activity (sometimes called twitching) and/or sudden increases in sympathetic activity. Tonic REM sleep is the portion of REM sleep that exists between the phasic bursts, in which low muscle tone is consistent [6]. REM sleep has typically been associated with vivid dreaming, based on early studies in which patients were awoken out of REM sleep. Although REM sleep accounts for less than a quarter of total sleep time (ranging from 18 to 23 percent), the function of this stage of sleep is still an area of debate.

During the first two decades of life the ratio between sleep and wakefulness changes dramatically. The amount of sleep during a 24-hour period decreases and the ratio between REM sleep and NREM sleep changes; the amount of REM sleep decreases with increasing age. Within NREM sleep, the age-related changes mainly affect the Slow Wave Sleep (N3) [7]. Another sleep related change from the ontogenetic perspective is the development of the sleep-wake-cyclicity during a 24-h day. This develops from multiple sleep phases during the day in infancy into two distinct sleep phases (night sleep and a nap in the afternoon in 
Kindergarten children), to one sleep phase during the night in school-age children (in most societies). With development, the architecture of sleep during the night changes. The number of sleep cycles decreases, the length of cycles increases and many variables characterizing sleep macrostructure show age related changes. REM sleep latency, sleep efficiency index, stage N2 and number of stage shifts increase with age, while total sleep time, wake after sleep onset, stage N3, and REM decrease with age [8]. These changes in sleep architecture have shown relatively low sex dependent differences. Aside from physiological changes there are also cultural and sociological influences on the sleep patterns over the developmental years, mainly regarding the amount of sleep. In a meta-analysis based on data from 23 countries, results indicate that sleep time varies between school days and non-school days. On non-school days, time in bed is longer in all age groups. In 9 to 18-year-old children and adolescents, sleep time declines with age by an amount of 14 minutes a day, per year of age, on school days and by 7 minutes on weekend days, while large differences in sleep habits and rituals exists between countries and societies [9].

Sleep difficulties are common in infants and children. About $20 \%-30 \%$ of children aged 1 - 3 years, experience sleep problems; frequent night awakenings and insufficient sleep duration are most commonly reported [10]. Pediatric sleep problems were shown to adversely influence children's health-related outcomes such as growth, body mass index, and blood pressure [11]. In adults, the risk for health-related problems, both physical and mental, show a U-shape curve pattern displaying more illness in the very short and very long sleepers [12] [13] [14] [15].

A population-based study in 1724 adolescents aged $10-19$ years reported both short and long sleep duration to be associated with lower academic performance scores [16], whereas lack of consolidated sleep (e.g., frequent awakenings) in 6 - 36 months infants, a proxy for maturation of sleep patterns, has been related to deficits in neurobehavioral tasks (e.g. reaction time, sustained attention, and working memory). Larger cohorts have not only pin-pointed a rather high incidence of sleep disturbances in children (20\% - 30\%) [17] but also a strong association between disturbed sleep and learning difficulties as well as diminished academic success [18] [19] [20].

In a recent study on 2800 children in the Netherlands [21], sleep and other developmental measurements were recorded by maternal interviews and questionnaires at 24 months and intelligence exams (both verbal and nonverbal) were conducted at the age of 6 . It was shown that sleep fragmentation at 24 months (mainly frequent awakenings i.e. $>3$ times per night), were independently associated with lower nonverbal intelligence, but not with verbal comprehension. Furthermore, the data suggests that both short $(<11$ hours in 24 -hour period) and long sleep duration ( $>14$ hours in 24-hour period) in 24-month-old toddlers were risk factors for unfavorable cognitive outcomes at 6 years of age [21]. 


\section{The Role of Non-REM Sleep on Brain Development}

Sleep is a homeostatic process that is assumed to support the brain's information processing in two different ways. Firstly, the encoding of information during wakefulness leads to widespread synaptic potentiation in neuronal networks, sleep mediates a global synaptic downscaling and renormalization of these networks, thereby renewing the brain's encoding capacity for the learning of new information [22] [23]. Secondly, sleep supports the formation and consolidation of long-term memories [24] [25]. More specifically, sleep aids the episodic memory system that encodes consciously experienced events into explicit memory and thereby essentially relies on circuitry connecting the hippocampus and prefrontal cortical networks [26] [27]. Of the EEG phenomena that characterize sleep, slow waves activity (SWA) and sleep spindles have been mostly used to characterize brain maturation. Both of these oscillatory phenomena occur during Non-REM sleep and notably, both phenomena have also been related to synaptic plasticity processes underlying memory consolidation during sleep [28] [29] [30]. In most mammalian species studied in detail, sleep duration is highest during the neonatal period, the phase of life that is characterized by rapid brain development and synaptic plasticity [31] [32] [33]. Synaptic plasticity during development has been correlated in single neuron recording studies in animal models; In Vitro studies have shown Long-Term Synaptic Potentiation (LTP) and Long-Term Synaptic Depression (LTD) [reviewed in [34] [35]]. LTP and LTD refer to use dependent, persistent alterations in synaptic weights that strengthen or weaken, specific synapses respectively [36]. Specifically, the increase in SWA after periods of extended wakefulness is assumed to reflect a global network increase in synaptic strength (mainly as an increase in LTP) because of extended information uptake into these networks. The decrease in SWA across subsequent sleep reflects downscaling in global synaptic strength (mainly with LTD recording being more prominent) [23]. The changes in SWA characterizing early development appear to be, in much the same way, paralleled by changes in synaptic connectivity. Global synaptic connectivity shows an overwhelming increase during the initial years of life, reaching a plateau around puberty, before it starts to decrease during adolescence. Unlike synaptogenesis, other indicators of brain maturation, such as fiber myelinization, show mostly linear rather than inverted $U$-shaped trajectories across childhood and adolescence, suggesting that the developmental link to SWA is specific to the formation of synaptic connectivity [37]. Cortical maturation is reflected in changes of the sleep electroencephalogram (EEG), specifically within nonrapid eye movement (NREM) sleep [37]-[43]. Dominant brain oscillations during NREM sleep, slow wave activity (SWA) and sleep spindles, have been associated with performance on measures of cognitive performance, "IQ" (e.g. children 7 - 11 years of age who had lower sleep spindle frequency displayed better performance on the perceptual reasoning and working memory in Wechsler Intelligence Scale for Children-IV (WISC-IV)) [44]-[49], learning efficiency [50] [51], memory con- 
solidation [25] [52] [53] [54] [55] [56], and motor skill development (e.g. in a study of 30 primary school children asked to complete finger sequence tapping tasks in a repeated-measures design, spanning 4 days, children performed better if they had less slow spindles, more fast spindles and faster slow waves) [41] [57] [58].

SWA further likely reflects synaptic density because more and stronger synapses benefit synchronization [59] [60]. SWA changes dramatically during development, peaking before puberty and gradually declining thereafter. These maturation dynamics follow the trajectory of cortical development, such as the formation and pruning of synapses [61] [62].

The presence of NREM sleep regulation in both neonatal rats [63] and humans [64] [65] suggests that NREM sleep may be important for all developing animals. The maturation of NREM sleep not only coincides with the formation of thalamocortical and intracortical patterns of innervation and periods of heightened synaptogenesis, but it is also associated with important processes in synaptic remodeling [66] [67].

During NREM sleep, waking patterns of neuronal activity are reactivated, suggesting that information acquired during wakefulness is further processed during this sleep state. Buzsaki et al. (1996) suggested that sharp wave bursts initiated in the hippocampus during slow-wave sleep (SWS) and associated with theta and gamma oscillations may provide the mechanism by which "quanta" of information may be relayed back to the neocortex during memory consolidation [68]. His group further demonstrated a correlation between neocortical and hippocampal activity during SWS, which suggests that these hippocampal patterns are coupled selectively to the neocortical cell groups that participate in the triggering of the bursts [29]. It is therefore possible that NREM sleep contributes to synaptic remodeling by providing an endogenous source of repetitive, synchronized activity within specific neuronal pathways [69].

Slow waves peak regionally over occipital regions in 2- to 5-year-old children but show a clear maximum over frontal regions in adults. The underlying shift from more posterior to frontal regions may reflect the development of more sophisticated cognitive processes that children develop with age [41]. Sleep spindles $(10-16 \mathrm{~Hz})$ consisting of a waxing and waning of the EEG lasting between 0.5 and $2 \mathrm{~s}$ [70] represent thalamocortical derived activity [71] and also reflect maturational and regional differences during NREM sleep and are observed in infants as young as $1-2$ months [72]. Slow $(11-13 \mathrm{~Hz})$ and fast spindles (13 $16 \mathrm{~Hz}$ ) are formed at around 24 months [73], slower frequencies are located over frontal regions and are more pronounced during slow wave sleep, whereas faster frequencies are located over centroparietal regions and are more prominent during stage 2 sleep [58] [71] [74].

There are also remarkable changes in sleep spindle characteristics in the first few months of life [75] [76] [77] [78] [79]. Alterations of sleep spindle characteristics are thought to reflect the maturation of the thalamocortical system [71]. 
Slow wave activity which represents $\delta$ power band during NREM sleep, has been shown to regionally mature from toddlerhood to adulthood from posterior to anterior brain regions closely mirroring cortical maturation, specifically grey matter [39] [40] [57] [80].

In a study measuring EEG in daytime naps of thirty healthy 12 - 30 months-old infants that completed developmental assessment (Mullen Early Scales of Learning (MSEL) and the Vineland Adaptive Behavior Scales-2nd Edition (VABS-2)), low $\delta$ waves and high $\theta$ were specifically predictive for skill maturation, whereas general low activity seemed to increase gradually in the frontal areas of the brain with age [81]. It was shown that absolute $\delta$ power increases globally before puberty and thereafter consistently decreases with age. The anabolic effects of Slow Wave Sleep (SWS) have been described in conjunction with the peak in Growth Hormone $(\mathrm{GH})$ secretion in the first two hours of sleep, mainly during SWS [82].

Studies in both infants and children have revealed robust effects of sleep benefiting hippocampus-dependent memory consolidation. As a prominent characteristic, sleep-dependent memory consolidation during early development is expressed particularly in the transformation of hippocampus-dependent representations, such that the abstraction of invariant and generalized memories is facilitated, rather than in stabilizing representations in the form in which they were encoded. Thus, in infants, sleep supports the abstraction of grammatical rules from a learned artificial language rather than strengthening the representations of the words. In children, the most profound effect of sleep was on the abstraction of explicit knowledge from implicitly trained materials [37].

Synaptic connectivity assumes a leading role for the prefrontal-hippocampal memory system that during wakefulness rapidly encodes episodes, whereby the hippocampus keeps the episodic nature of the representation by binding an experienced event into its unique spatiotemporal context. During subsequent SWS, repeated neuronal reactivation of newly encoded representations (or parts of them) in hippocampal networks is thought to promote a transformation of these representations such that they become largely redistributed to extrahippocampal networks (mainly neocortical and striatal networks), which serve as long-term stores [26].

As the hippocampal-frontal connectivity mainly correlates with a development of semantic and lexical memory and in contrast to procedural tasks, children between 7 and 14 years of age have been found to exhibit robust sleep-dependent consolidation on various declarative tasks like word-pair learning, lexical integration of novel words, and emotional episodic memory tasks [55] [83] [84] [85] [86] [87]. The presence of competitive interactions between hippocampus-dependent and procedural memory systems during sleep-dependent consolidation is also suggested by studies in children with attention deficit hyper-activity disorder (ADHD) [88] [89] [90]. Children diagnosed with ADHD displayed distinct overnight gains in finger sequence-tapping skill compared to healthy controls 
but with impaired sleep-dependent consolidation on declarative types of task. Children with ADHD are characterized by a malfunction of the prefrontal cortex and exhibit a less mature topography of SWA [91]. It has been speculated that their slow oscillations, specifically those originating from the prefrontal cortex, are dysfunctional (or not yet matured) in controlling the consolidation of hippocampus-dependent memory and the accompanying interactions with other memory systems [92] [93].

As in adults, hippocampus-dependent memory consolidation in children has been linked to SWS and slow oscillations. Some studies in children revealed positive correlations between overnight consolidation of hippocampus-dependent memory and slow oscillation power or measures of spindle activity during post-learning Non-REM sleep [50] [51] [55] [88]. However, in a direct comparison between children and adults, the magnitude of the sleep effect on the consolidation of word-pair associations appeared to be of similar magnitude in both age groups, although in these experiments the children showed an approximately twofold greater amount of SWS than the adults [83].

This view is corroborated by a study that tested the effect of sleep in 8 11-year-old children and adults, on the abstraction of explicit knowledge from an implicitly trained motor task, the "button-box task" (which is a coarse motor version of a serial-reaction task) [94]. When asked immediately after training or after a retention period of daytime wakefulness, the children had only little explicit knowledge about the trained sequence. However, when asked after a night of sleep, the children had developed almost perfect explicit sequence knowledge, with this specific increase in explicit knowledge being strikingly more pronounced than in adults. Notably, in both children and adults, sleep-dependent gains in explicit sequence knowledge were associated with increased SWA during Non-REM sleep.

Sleep supports the consolidation of memory from early on and this consolidation appears to be closely linked to signs of SWS. Compared with adults, the sleep-dependent consolidation of hippocampus-dependent memory and its link to SWS is expressed most prominently in tasks requiring the abstraction of new schemata and invariants, rather than in a mere stabilization of representations in the same form in which they were encoded. Thus, children outperform adults in generating explicit sequence knowledge from an implicitly trained serial reaction-time task [94] whereas the benefit from sleep for paired word associations is equivalent to that in adults. Additionally, sleep in infants even seems selectively to support the abstraction of grammatical rules from sentences of an artificial language while simultaneously speeding the forgetting of single words presented during encoding [95]. In a study [96] of one hundred sixty two 6 - 8 months old infants, the infants were exposed to new words as labels for new object categories. In the memory test about an hour later, generalization to novel category exemplars was tested with quantified EEG measuring the response. The infants differed in the amount of sleep they usually spend during daytime napping. The study showed that after a longer nap ( $\sim 50$ minutes), infants reached a higher 
developed stage than after a short nap ( $\sim 30$ minutes). The observed effect of sleep time on the quality of memory was linked only to the duration of NREM stage 2 (N2). The more time an infant spent in N2, the fewer EEG markers existed for perceptual associative memory alone, and the greater EEG markers indicating genuine lexical-semantic memory. It seems that the consolidation of object-word pairings during sleep parallels developmental stages of lexical memory formation. These new findings suggest that sleep-dependent system consolidation during development plays an essential role in establishing entire memory systems with separate stores for longer-term memories in different domains.

\section{The Role of REM Sleep on Brain Development}

It seems paradoxical that CNS maturational processes in the late prenatal and neonatal periods are highly active at the time that extrinsic sensory stimulation is quite limited. During these periods, a large percentage of time is spent in REM sleep, characterized by endogenous, intense, generalized neuronal firing in most areas of the brain [97], consequently it was suggested by early investigators that the primary purpose of REM sleep was to act as an inducer of CNS development in the fetus and the neonate [33]. Based on the early myelination of the sensory processing areas in the CNS, it was further proposed that REM sleep provided endogenous stimulation to these areas. Studies of REM sleep deprivation have provided consistent support to the role of REM sleep in brain maturation and especially on subsequent visual development [98]-[103].

Kittens with normal binocular vision subjected to REM sleep deprivation during the second week of a 2-week monocular deprivation (MD) period (blindfolding one eye), had further anatomically and functionally deleterious effects on the lateral geniculate nucleus (LGN) relative to MD alone [98]. Further, it was demonstrated that the elimination of ponto-geniculo-occipital wave phasic activity during REM sleep (a method that preserves sleep and wake proportions as well as other REM features) similarly yielded enhanced plasticity effects in the LGN [101], and REM sleep deprivation delayed the development of synaptic plasticity in the LGN [102] and retarded the maturational reduction of long-term potentiation (LTP) in visual cortex of immature rats [103], as was the case for rats reared in darkness [104].

These results emphasize that REM sleep is also an important part of visual development after birth, stimulating neurons in a fundamentally different way from that derived from visual experience [105]. Moreover, since REM sleep excites neural components elsewhere in the brain, not just in the visual system, it also might help other areas of the brain develop.

Studies in rats show that 1 week of REM sleep deprivation prolongs the critical period for the developmentally regulated form of LTP in vitro [103]. That is, after REM sleep deprivation, LTP can be induced at ages when this form of plasticity is normally no longer present. A similar extension of the critical period was not seen in cortical slices from control rats. Conversely, REM sleep deprivation 
had no effect on a non-developmentally regulated form of LTP evoked by layer IV stimulation. Subsequent studies from these investigators showed that this plasticity could be partially rescued if REM sleep deprivation was administered near (or overlapping) the end of the critical period [106] [107].

More recent findings show that the effects of REM sleep deprivation can be prevented by chronically infusing brain-derived neurotrophic factor (BDNF) into the visual cortex. This indicates that REM sleep may normally promote BDNF synthesis [108].

A large number of studies also show that in vitro hippocampal LTP (either the incidence or maintenance) is reduced in rodents that undergo varying amounts of REM sleep deprivation, total sleep deprivation, or sleep restriction [109].

The underlying mechanisms mediating the effects of sleep loss on LTP and LTD are not well understood. However, they do not appear to be simply due to indirect effects of the sleep deprivation procedures, these effects can be related to changes in stress hormones, decrements in hippocampal NMDA receptor function and ERK/MAPK activation possibly in combination with reductions in plasticity-related mRNAs or proteins and elevated concentrations of PDE4 and extracellular adenosine [109].

REM sleep deprived animals have a reduced brain size, hyperactivity, anxiety, attention and learning difficulties, increased voluntary alcohol consumption and reduced masculine sexual behavior. Moreover, while environmental enrichment has been shown to enhance cortical maturation, this was no longer possible in the REM sleep deprived rats [110].

The mounting empirical evidence from both animal model and in vitro studies points to a massive importance of REM sleep in maturation of neuronal systems, mostly in the visual cortex but also in more frontal cortical areas.

\section{Conclusion}

"Sleep is of the brain, by the brain and for the brain" [111] and it seems that even though much more research is required in order to further the understanding of the role of sleep in brain and bodily development, research so far has shown the vital role that sleep, in its different aspects, plays a decisive role in brain development, especially in early life. It is therefore no wonder that in the first decade of life, where the rate of growth and development is at its peak, the child should spend half of the day and sometimes more, sleeping. This understanding should light the way to all who take part in childcare towards viewing sleep not merely as a physiological necessity in the daily schedule, but as an active process of brain development, and by that, facilitating sleep promoting in children and adolescents and ensuring that the needed amount of time and sleep environment has an honorable place in the daily schedule.

\section{Conflicts of Interest}

The authors declare no conflicts of interest regarding the publication of this paper. 


\section{References}

[1] Danker-Hopfe, H. (2011) Growth and Development of Children with a Special Focus on Sleep. Progress in Biophysics \& Molecular Biology, 107, 333-338. https://doi.org/10.1016/j.pbiomolbio.2011.08.014

[2] Lodygensky, G.A., Vasung, L., Sizonenko, S.V. and Hüppi, P.S. (2010) Neuroimaging of Cortical Development and Brain Connectivity in Human Newborns and Animal Models. Journal of Anatomy, 217, 418-428. https://doi.org/10.1111/j.1469-7580.2010.01280.x

[3] WHO Multicentre Growth Reference Study Group (2007) WHO Child Growth Standards: Head Circumference-for-Age, Triceos Skinfold-for-Age and Subscapular Skinfold-for-Age: Methods and Development. World Health Organization, Geneva.

[4] Paus, T., Collins, D.L., Evans, A.C., Leonard, G., Pike, B. and Zijdenbos, A. (2001) Maturation of White Matter in the Human Brain: A Review of Magnetic Resonance Studies. Brain Research Bulletin, 54, 255-266.

https://doi.org/10.1016/S0361-9230(00)00434-2

[5] Ohayon, M.M., Carskadon, M.A., Guilleminault, C. and Vitiello, M.V. (2004) Meta-Analysis of Quantitative Sleep Parameters from Childhood to Old Age in Healthy Individuals: Developing Normative Sleep Values across the Human Lifespan. Sleep, 27, 1255-1273. https://doi.org/10.1093/sleep/27.7.1255

[6] Berry, R., Quan, S. and Abreu, A. (2020) The AASM Manual for the Scoring of Sleep and Associated Events: Rules, Terminology and Technical Specifications, Version 2.6. American Academy of Sleep Medicine, Darien.

[7] Carskadon, W.C. and Dement, M.A. (2011) Normal Human Sleep: An Overview. In: Principles and Practice of Sleep Medicine, Fifth Edition, Elsevier Saunders, St. Louis, 16-26. https://doi.org/10.1016/B978-1-4160-6645-3.00002-5

[8] Scholle, S., et al. (2011) Normative Values of Polysomnographic Parameters in Childhood and Adolescence: Quantitative Sleep Parameters. Sleep Medicine, 12, 542-549. https://doi.org/10.1016/j.sleep.2010.11.011

[9] Olds, T., Blunden, S., Petkov, J. and Forchino, F. (2010) The Relationships between Sex, Age, Geography and Time in Bed in Adolescents: A Meta-Analysis of Data from 23 Countries. Sleep Medicine Reviews, 14, 371-378.

https://doi.org/10.1016/j.smrv.2009.12.002

[10] Sadeh, A., Mindell, J.A., Luedtke, K. and Wiegand, B. (2009) Sleep and Sleep Ecology in the First 3 Years: A Web-Based Study. Journal of Sleep Research, 18, 60-73. https://doi.org/10.1111/j.1365-2869.2008.00699.x

[11] Nixon, G.M., et al. (2008) Short Sleep Duration in Middle Childhood: Risk Factors and Consequences. Sleep, 31, 71-78. https://doi.org/10.1093/sleep/31.1.71

[12] Chaput, J.P., et al. (2011) Short Sleep Duration Is Independently Associated with Overweight and Obesity in Quebec Children. Canadian Journal of Public Health, 102, 369-374. https://doi.org/10.1007/BF03404179

[13] Spruyt, K., Molfese, D.L. and Gozal, D. (2011) Sleep Duration, Sleep Regularity, Body Weight, and Metabolic Homeostasis in School-Aged Children. Pediatrics, 127, e345-e352. https://doi.org/10.1542/peds.2010-0497

[14] Leng, Y., et al. (2015) Sleep Duration and Risk of Fatal and Nonfatal Stroke: A Prospective Study and Meta-Analysis. Neurology, 84, 1072-1079. https://doi.org/10.1212/WNL.0000000000001371

[15] van den Berg, J.F., et al. (2008) Actigraphic Sleep Duration and Fragmentation Are Related to Obesity in the Elderly: The Rotterdam Study. International Journal of 
Obesity, 32, 1083-1090. https://doi.org/10.1038/ijo.2008.57

[16] Eide, E.R. and Showalter, M.H. (2012) Sleep and Student Achievement. Eastern Economic Journal, 38, 512-524. https://doi.org/10.1057/eej.2011.33

[17] Mindell, J.A., Owens, J.A. and Carskadon, M.A. (1999) Developmental Features of Sleep. Child and Adolescent Psychiatric Clinics of North America, 8, 695-725. https://doi.org/10.1016/S1056-4993(18)30149-4

[18] Carskadon, M.A., Acebo, C. and Jenni, O.G. (2004) Regulation of Adolescent Sleep: Implications for Behavior. Annals of the New York Academy of Sciences, 1021, 276-291. https://doi.org/10.1196/annals.1308.032

[19] Urschitz, M.S., et al. (2003) Snoring, Intermittent Hypoxia and Academic Performance in Primary School Children. American Journal of Respiratory and Critical Care Medicine, 168, 464-468. https://doi.org/10.1164/rccm.200212-1397OC

[20] Halbower, A.C. and Mahone, E.M. (2006) Neuropsychological Morbidity Linked to Childhood Sleep-Disordered Breathing. Sleep Medicine Reviews, 10, 97-107. https://doi.org/10.1016/j.smrv.2005.10.002

[21] Kocevska, D., et al. (2017) Early Childhood Sleep Patterns and Cognitive Development at Age 6 Years: The Generation R Study. Journal of Pediatric Psychology, 42, 260-268. https://doi.org/10.1093/jpepsy/jsv168

[22] Tononi, G. and Cirelli, C. (2006) Sleep Function and Synaptic Homeostasis. Sleep Medicine Reviews, 10, 49-62. https://doi.org/10.1016/j.smrv.2005.05.002

[23] Tononi, G. and Cirelli, C. (2014) Sleep and the Price of Plasticity: From Synaptic and Cellular Homeostasis to Memory Consolidation and Integration. Neuron, 81, 12-34. https://doi.org/10.1016/j.neuron.2013.12.025

[24] Diekelmann, S. and Born, J. (2010) The Memory Function of Sleep. Nature Reviews Neuroscience, 11, 114-126. https://doi.org/10.1038/nrn2762

[25] Rasch, B. and Born, J. (2013) About Sleep's Role in Memory. Physiological Reviews, 93, 681-766. https://doi.org/10.1152/physrev.00032.2012

[26] Inostroza, M. and Born, J. (2013) Sleep for Preserving and Transforming Episodic Memory. Annual Review of Neuroscience, 36, 79-102.

https://doi.org/10.1146/annurev-neuro-062012-170429

[27] Battaglia, F.P., Benchenane, K., Sirota, A., Pennartz, C.M.A. and Wiener, S.I. (2011) The Hippocampus: Hub of Brain Network Communication for Memory. Trends in Cognitive Sciences, 15, 310-318. https://doi.org/10.1016/j.tics.2011.05.008

[28] Timofeev, I., Grenier, F., Bazhenov, M., Houweling, A.R., Sejnowski, T.J. and Steriade, M. (2002) Short- and Medium-Term Plasticity Associated with Augmenting Responses in Cortical Slabs and Spindles in Intact Cortex of Cats in Vivo. The Journal of Physiology, 542, 583-598. https://doi.org/10.1113/jphysiol.2001.013479

[29] Sirota, A., Csicsvari, J., Buhl, D. and Buzsáki, G. (2003) Communication between Neocortex and Hippocampus during Sleep in Rodents. Proceedings of the National Academy of Sciences of the United States of America, 100, 2065-2069. https://doi.org/10.1073/pnas.0437938100

[30] Chauvette, S., Seigneur, J. and Timofeev, I. (2012) Sleep Oscillations in the Thalamocortical System Induce Long-Term Neuronal Plasticity. Neuron, 75, 1105-1113. https://doi.org/10.1016/j.neuron.2012.08.034

[31] Frank, M.G. and Heller, H.C. (1997) Development of REM and Slow Wave Sleep in the Rat. American Journal of Physiology, 272, R1792-R1799. https://doi.org/10.1152/ajpregu.1997.272.6.R1792

[32] Jouvet-Mounier, D., Astic, L. and Lacote, D. (1970) Ontogenesis of the States of 
Sleep in Rat, Cat, and Guinea Pig during the First Postnatal Month. Developmental Psychobiology, 2, 216-239. https://doi.org/10.1002/dev.420020407

[33] Roffwarg, H.P., Muzio, J.N. and Dement, W.C. (1966) Ontogenetic Development of the Human Sleep-Dream Cycle. Science, 152, 604-619.

https://doi.org/10.1126/science.152.3722.604

[34] Benington, J.H. and Frank, M.G. (2003) Cellular and Molecular Connections between Sleep and Synaptic Plasticity. Progress in Neurobiology, 69, 71-101. https://doi.org/10.1016/S0301-0082(03)00018-2

[35] Frank, M.G. and Benington, J.H. (2006) The Role of Sleep in Memory Consolidation and Brain Plasticity: Dream or Reality? Neuroscientist, 12, 477-488. https://doi.org/10.1177/1073858406293552

[36] Malenka, R.C. and Bear, M.F. (2004) LTP and LTD: An Embarrassment of Riches. Neuron, 44, 5-21. https://doi.org/10.1016/j.neuron.2004.09.012

[37] Huber, R. and Born, J. (2014) Sleep, Synaptic Connectivity, and Hippocampal Memory during Early Development. Trends in Cognitive Sciences, 18, 141-152. https://doi.org/10.1016/j.tics.2013.12.005

[38] Novelli, L., et al. (2016) Mapping Changes in Cortical Activity during Sleep in the First 4 Years of Life. Journal of Sleep Research, 25, 381-389. https://doi.org/10.1111/jsr.12390

[39] Buchmann, A., et al. (2011) EEG Sleep Slow-Wave Activity as a Mirror of Cortical Maturation. Cerebral Cortex, 21, 607-615. https://doi.org/10.1093/cercor/bhq129

[40] Kurth, S., Ringli, M., Geiger, A., LeBourgeois, M., Jenni, O.G. and Huber, R. (2010) Mapping of Cortical Activity in the First Two Decades of Life: A High-Density Sleep Electroencephalogram Study. Journal of Neuroscience, 30, 13211-13219. https://doi.org/10.1523/JNEUROSCI.2532-10.2010

[41] Kurth, S., et al. (2012) Mapping the Electrophysiological Marker of Sleep Depth Reveals Skill Maturation in Children and Adolescents. Neuroimage, 63, 959-965. https://doi.org/10.1016/j.neuroimage.2012.03.053

[42] Campbell, I.G. and Feinberg, I. (2009) Longitudinal Trajectories of Non-Rapid Eye Movement Delta and Theta EEG as Indicators of Adolescent Brain Maturation. Proceedings of the National Academy of Sciences of the United States of America, 106, 5177-5180. https://doi.org/10.1073/pnas.0812947106

[43] Ringli, M. and Huber, R. (2011) Developmental Aspects of Sleep Slow Waves: Linking Sleep, Brain Maturation and Behavior. Progress in Brain Research, 193, 63-82. https://doi.org/10.1016/B978-0-444-53839-0.00005-3

[44] Doucette, M.R., Kurth, S., Chevalier, N., Munakata, Y. and LeBourgeois, M.K. (2015) Topography of Slow Sigma Power during Sleep Is Associated with Processing Speed in Preschool Children. Brain Sciences, 5, 494-508. https://doi.org/10.3390/brainsci5040494

[45] Tessier, S., Lambert, A., Chicoine, M., Scherzer, P., Soulières, I. and Godbout, R. (2015) Intelligence Measures and Stage 2 Sleep in Typically-Developing and Autistic Children. International Journal of Psychophysiology, 97, 58-65. https://doi.org/10.1016/j.ijpsycho.2015.05.003

[46] Geiger, A., Huber, R., Kurth, S., Ringli, M., Jenni, O.G. and Achermann, P. (2011) The Sleep EEG as a Marker of Intellectual Ability in School Age Children. Sleep, 34, 181-189. https://doi.org/10.1093/sleep/34.2.181

[47] Fogel, S.M. and Smith, C.T. (2011) The Function of the Sleep Spindle: A Physiological Index of Intelligence and a Mechanism for Sleep-Dependent Memory Consoli- 
dation. Neuroscience \& Biobehavioral Reviews, 35, 1154-1165. https://doi.org/10.1016/j.neubiorev.2010.12.003

[48] Bódizs, R., Gombos, F., Ujma, P.P. and Kovács, I. (2014) Sleep Spindling and Fluid Intelligence across Adolescent Development: Sex Matters. Frontiers in Human Neuroscience, 8, Article 952. https://doi.org/10.3389/fnhum.2014.00952

[49] Gruber, R., et al. (2013) The Association between Sleep Spindles and IQ in Healthy School-Age Children. International Journal of Psychophysiology, 89, 229-240. https://doi.org/10.1016/j.ijpsycho.2013.03.018

[50] Lustenberger, C., Maric, A., Dürr, R., Achermann, P. and Huber, R. (2012) Triangular Relationship between Sleep Spindle Activity, General Cognitive Ability and the Efficiency of Declarative Learning. PLOS ONE, 7, e49561.

https://doi.org/10.1371/journal.pone.0049561

[51] Hoedlmoser, K., et al. (2014) Slow Sleep Spindle Activity, Declarative Memory, and General Cognitive Abilities in Children. Sleep, 37, 1501-1512. https://doi.org/10.5665/sleep.4000

[52] Lustenberger, C., Wehrle, F., Tüshaus, L., Achermann, P. and Huber, R. (2015) The Multidimensional Aspects of Sleep Spindles and Their Relationship to Word-Pair Memory Consolidation. Sleep, 38, 1093-1103. https://doi.org/10.5665/sleep.4820

[53] Marshall, L., Helgadóttir, H., Mölle, M. and Born, J. (2006) Boosting Slow Oscillations during Sleep Potentiates Memory. Nature, 444, 610-613. https://doi.org/10.1038/nature05278

[54] Lustenberger, C., Boyle, M.R., Alagapan, S., Mellin, J.M., Vaughn, B.V. and Fröhlich, F. (2016) Feedback-Controlled Transcranial Alternating Current Stimulation Reveals a Functional Role of Sleep Spindles in Motor Memory Consolidation. Current Biology, 26, 2127-2136. https://doi.org/10.1016/j.cub.2016.06.044

[55] Kurdziel, L., Duclos, K. and Spencer, R.M.C. (2013) Sleep Spindles in Midday Naps Enhance Learning in Preschool Children. Proceedings of the National Academy of Sciences of the United States of America, 110, 17267-17272. https://doi.org/10.1073/pnas.1306418110

[56] Ngo, H.V.V., Martinetz, T., Born, J. and Mölle, M. (2013) Auditory Closed-Loop Stimulation of the Sleep Slow Oscillation Enhances Memory. Neuron, 78, 545-553. https://doi.org/10.1016/j.neuron.2013.03.006

[57] Lustenberger, C., et al. (2017) Developmental Trajectories of EEG Sleep Slow Wave Activity as a Marker for Motor Skill Development during Adolescence: A Pilot Study. Developmental Psychobiology, 59, 5-14. https://doi.org/10.1002/dev.21446

[58] Astill, R.G., et al. (2014) Sleep Spindle and Slow Wave Frequency Reflect Motor Skill Performance in Primary School-Age Children. Frontiers in Human Neuroscience, 8, 910. https://doi.org/10.3389/fnhum.2014.00910

[59] Esser, S.K., Hill, S.L. and Tononi, G. (2007) Sleep Homeostasis and Cortical Synchronization: I. Modeling the Effects of Synaptic Strength on Sleep Slow Waves. Sleep, 30, 1617-1630. https://doi.org/10.1093/sleep/30.12.1617

[60] Vyazovskiy, V.V., et al. (2009) Cortical Firing and Sleep Homeostasis. Neuron, 63, 865-878. https://doi.org/10.1016/j.neuron.2009.08.024

[61] Peter, H.R. (1979) Synaptic Density in Human Frontal Cortex-Developmental Changes and Effects of Aging. Brain Research, 163, 195-205.

https://doi.org/10.1016/0006-8993(79)90349-4

[62] Huttenlocher, P.R. and Dabholkar, A.S. (1997) Regional Differences in Synaptogenesis in Human Cerebral Cortex. Journal of Comparative Neurology, 387, 167-178. 
https://doi.org/10.1002/(SICI)1096-9861(19971020)387:2<167::AID-CNE1>3.0.CO; $\underline{2-Z}$

[63] Alföldi, P., Tobler, I. and Borbély, A.A. (1990) Sleep Regulation in Rats during Early Development. American Journal of Physiology, 258, R634-R644. https://doi.org/10.1152/ajpregu.1990.258.3.R634

[64] Anders, T.F. and Roffwarg, H.P. (1973) The Effects of Selective Interruption and Deprivation of Sleep in the Human Newborn. Developmental Psychobiology, 6, 77-89. https://doi.org/10.1002/dev.420060110

[65] Thomas, D.A., et al. (1996) The Effect of Sleep Deprivation on Sleep States, Breathing Events, Peripheral Chemoresponsiveness and Arousal Propensity in Healthy 3 Month Old Infants. European Respiratory Journal, 9, 932-938. https://doi.org/10.1183/09031936.96.09050932

[66] Bear, M.F. and Malenka, R.C. (1994) Synaptic Plasticity: LTP and LTD. Current Opinion in Neurobiology, 4, 389-399. https://doi.org/10.1016/0959-4388(94)90101-5

[67] Cramer, K.S. and Sur, M. (1995) Activity-Dependent Remodeling of Connections in the Mammalian Visual System. Current Opinion in Neurobiology, 5, 106-111. https://doi.org/10.1016/0959-4388(95)80094-8

[68] Buzsáki, G. (1996) The Hippocampo-Neocortical Dialogue. Cerebral Cortex, 6, 81-92. https://doi.org/10.1093/cercor/6.2.81

[69] Kavanau, J.L. (1994) Sleep and Dynamic Stabilization of Neural Circuitry: A Review and Synthesis. Behavioural Brain Research, 63, 111-126. https://doi.org/10.1016/0166-4328(94)90082-5

[70] Gibbs, E.L. and Gibbs, F.A. (1941) Atlas of Electroencephalography. Boston City Hospital.

[71] De Gennaro, L. and Ferrara, M. (2003) Sleep Spindles: An Overview. Sleep Medicine Reviews, 7, 423-440. https://doi.org/10.1053/smrv.2002.0252

[72] Ellingson, R.J. (1982) Development of Sleep Spindle Bursts during the First Year of Life. Sleep, 5, 39-46. https://doi.org/10.1093/sleep/5.1.39

[73] Jankel, W.R. and Niedermeyer, E. (1985) Sleep Spindles. Journal of Clinical Neurophysiology, 2, 1-36. https://doi.org/10.1097/00004691-198501000-00001

[74] Andrillon, T., et al. (2011) Sleep Spindles in Humans: Insights from Intracranial EEG and Unit Recordings. Journal of Neuroscience, 31, 17821-17834. https://doi.org/10.1523/JNEUROSCI.2604-11.2011

[75] Jenni, O.G., Borbély, A.A. and Achermann, P. (2004) Development of the Nocturnal Sleep Electroencephalogram in Human Infants. American Journal of Physiology-Regulatory, Integrative and Comparative Physiology, 286, R528-R538. https://doi.org/10.1152/ajpregu.00503.2003

[76] Hughes, J.R. (1996) Development of Sleep Spindles in the First Year of Life. Clinical EEG and Neuroscience, 27, 107-115. https://doi.org/10.1177/155005949602700303

[77] Tanguay, P.E., Ornitz, E.M., Kaplan, A. and Bozzo, E.S. (1975) Evolution of Sleep Spindles in Childhood. Electroencephalography and Clinical Neurophysiology, 38, 175-181. https://doi.org/10.1016/0013-4694(75)90227-8

[78] Louis, J., Zhang, J.X., Revol, M., Debilly, G. and Challamel, M.J. (1992) Ontogenesis of Nocturnal Organization of Sleep Spindles: A Longitudinal Study during the First 6 Months of Life. Electroencephalography and Clinical Neurophysiology, 83, 289-296. https://doi.org/10.1016/0013-4694(92)90088-Y

[79] Scholle, S., Zwacka, G. and Scholle, H.C. (2007) Sleep Spindle Evolution from In- 
fancy to Adolescence. Clinical Neurophysiology, 118, 1525-1531. https://doi.org/10.1016/j.clinph.2007.03.007

[80] Feinberg, I. and Campbell, I.G. (2010) Sleep EEG Changes during Adolescence: An Index of a Fundamental Brain Reorganization. Brain and Cognition, 72, 56-65. https://doi.org/10.1016/j.bandc.2009.09.008

[81] Page, J., Lustenberger, C. and Fr Hlich, F. (2018) Social, Motor, and Cognitive Development through the Lens of Sleep Network Dynamics in Infants and Toddlers between 12 and 30 Months of Age. Sleep, 41, 1-10. https://doi.org/10.1093/sleep/zsy024

[82] Shapiro, C. (1981) Growth Hormone-Sleep Interactions: A Review. In: Research Communication in Psychology, Psychiatry and Behavior. Growth Hormone-Sleep Interactions: A Review, PJD Publications Ltd., 6 No. 2.

[83] Wilhelm, I., Diekelmann, S. and Born, J. (2008) Sleep in Children Improves Memory Performance on Declarative But Not Procedural Tasks. Learning \& Memory, 15, 373-377. https://doi.org/10.1101/lm.803708

[84] Prehn-Kristensen, A., Göder, R., Chirobeja, S., Bressmann, I., Ferstl, R. and Baving, L. (2009) Sleep in Children Enhances Preferentially Emotional Declarative But Not Procedural Memories. Journal of Experimental Child Psychology, 104, 132-139. https://doi.org/10.1016/j.jecp.2009.01.005

[85] Henderson, L.M., Weighall, A.R., Brown, H. and Gaskell, M.G. (2012) Consolidation of Vocabulary Is Associated with Sleep in Children. Developmental Science, 15, 674-687. https://doi.org/10.1111/j.1467-7687.2012.01172.x

[86] Backhaus, J., Hoeckesfeld, R., Born, J., Hohagen, F. and Junghanns, K. (2008) Immediate as Well as Delayed Post Learning Sleep But Not Wakefulness Enhances Declarative Memory Consolidation in Children. Neurobiology of Learning and Memory, 89, 76-80. https://doi.org/10.1016/j.nlm.2007.08.010

[87] Potkin, K.T. and Bunney, W.E. (2012) Sleep Improves Memory: The Effect of Sleep on Long Term Memory in Early Adolescence. PLoS ONE, 7, e42191. https://doi.org/10.1371/journal.pone.0042191

[88] Prehn-Kristensen, A., et al. (2011) Reduced Sleep-Associated Consolidation of Declarative Memory in Attention-Deficit/Hyperactivity Disorder. Sleep Medicine, 12, 672-679. https://doi.org/10.1016/j.sleep.2010.10.010

[89] Prehn-Kristensen, A., et al. (2011) Sleep Restores Daytime Deficits in Procedural Memory in Children with Attention-Deficit/Hyperactivity Disorder. Research in Developmental Disabilities, 32, 2480-2488. https://doi.org/10.1016/j.ridd.2011.06.021

[90] Prehn-Kristensen, A., Munz, M., Molzow, I., Wilhelm, I., Wiesner, C.D. and Baving, L. (2013) Sleep Promotes Consolidation of Emotional Memory in Healthy Children But Not in Children with Attention-Deficit Hyperactivity Disorder. PLoS ONE, 8, e65098. https://doi.org/10.1371/journal.pone.0065098

[91] Ringli, M., Souissi, S., Kurth, S., Brandeis, D., Jenni, O.G. and Huber, R. (2013) Topography of Sleep Slow Wave Activity in Children with Attention-Deficit/Hyperactivity Disorder. Cortex, 49, 340-347. https://doi.org/10.1016/j.cortex.2012.07.007

[92] Wilhelm, I., Prehn-Kristensen, A. and Born, J. (2012) Sleep-Dependent Memory Consolidation-What Can Be Learnt from Children? Neuroscience \& Biobehavioral Reviews, 36, 1718-1728. https://doi.org/10.1016/j.neubiorev.2012.03.002

[93] Miano, S., Donfrancesco, R., Bruni, O., Ferri, R., Galiffa, S., Pagani, J., Montemitro, E., Kheirandish, L. and Gozal, D. (2006) NREM Sleep Instability Is Reduced in Children with Attention-Deficit/Hyperactivity Disorder. Sleep, 29, 797-803. 
[94] Wilhelm, I., Rose, M., Imhof, K.I., Rasch, B., Büchel, C. and Born, J. (2013) The Sleeping Child Outplays the Adult's Capacity to Convert Implicit into Explicit Knowledge. Nature Neuroscience, 16, 391-393. https://doi.org/10.1038/nn.3343

[95] Gómez, R.L., Bootzin, R.R. and Nadel, L. (2006) Naps Promote Abstraction in Language-Learning Infants. Psychological Science, 17, 670-674. https://doi.org/10.1111/j.1467-9280.2006.01764.x

[96] Friedrich, M., Wilhelm, I., Mölle, M., Born, J. and Friederici, A.D. (2017) The Sleeping Infant Brain Anticipates Development. Current Biology, 27, 2374-2380.e3. https://doi.org/10.1016/j.cub.2017.06.070

[97] Mirmiran, M. and Ariagno, R.L. (2009) Role of Rem Sleep in Brain Development and Plasticity. In: Sleep and Brain Plasticity, Oxford University Press, Oxford, 181-188. https://doi.org/10.1093/acprof:oso/9780198574002.003.0010

[98] Marks, G.A., Shaffery, J.P., Oksenberg, A., Speciale, S.G. and Roffwarg, H.P. (1995) A Functional Role for REM Sleep in Brain Maturation. Behavioural Brain Research, 69, 1-11. https://doi.org/10.1016/0166-4328(95)00018-O

[99] Oksenberg, A., Shaffery, J.P., Marks, G.A., Speciale, S.G., Mihailoff, G. and Roffwarg, H.P. (1996) Rapid Eye Movement Sleep Deprivation in Kittens Amplifies LGN Cell-Size Disparity Induced by Monoclonal Deprivation. Developmental Brain Research, 97, 51-61. https://doi.org/10.1016/S0165-3806(96)00131-9

[100] Shaffery, J.P., Oksenberg, A., Marks, G.A., Speciale, S.G., Mihailoff, G. and Roffwarg, H.P. (1998) REM Sleep Deprivation in Monocularly Occluded Kittens Reduces the Size of Cells in LGN Monocular Segment. Sleep, 21, 837-845.

https://doi.org/10.1093/sleep/21.8.837

[101] Shaffery, J.P., Roffwarg, H.P., Speciale, S.G. and Marks, G.A. (1999) Ponto-Geniculo-Occipital-Wave Suppression Amplifies Lateral Geniculate Nucleus Cell-Size Changes in Monocularly Deprived Kittens. Developmental Brain Research, 114, 109-119. https://doi.org/10.1016/S0165-3806(99)00027-9

[102] Hogan, D., Roffwarg, H.P. and Shaffery, J.P. (2001) The Effects of 1 Week of REM Sleep Deprivation on Parvalbumin and Calbindin Immunoreactive Neurons in Central Visual Pathways of Kittens. Journal of Sleep Research, 10, 285-296. https://doi.org/10.1046/j.1365-2869.2001.00270.x

[103] Shaffery, J.P., Sinton, C.M., Bissette, G., Roffwarg, H.P. and Marks, G.A. (2002) Rapid Eye Movement Sleep Deprivation Modifies Expression of Long-Term Potentiation in Visual Cortex of Immature Rats. Neuroscience, 110, 431-443. https://doi.org/10.1016/S0306-4522(01)00589-9

[104] Kirkwood, A., Lee, H.K. and Bear, M.F. (1995) Co-Regulation of Long-Term Potentiation and Experience-Dependent Synaptic Plasticity in Visual Cortex by Age and Experience. Nature, 375, 328-331. https://doi.org/10.1038/375328a0

[105] Marks, G.A., Roffwarg, H.P. and Shaffery, J.P. (1999) Neuronal Activity in the Lateral Geniculate Nucleus Associated with Ponto-Geniculo-Occipital Waves Lacks Lamina Specificity. Brain Research, 815, 21-28. https://doi.org/10.1016/S0006-8993(98)01008-7

[106] Shaffery, J.P., Lopez, J., Bissette, G. and Roffwarg, H.P. (2006) Rapid Eye Movement Sleep Deprivation Revives a Form of Developmentally Regulated Synaptic Plasticity in the Visual Cortex of Post-Critical Period Rats. Neuroscience Letters, 391, 96-101. https://doi.org/10.1016/j.neulet.2005.08.044

[107] Shaffery, J.P. and Roffwarg, H.P. (2003) Rapid Eye-Movement Sleep Deprivation Does Not "Rescue" Developmentally Regulated Long-Term Potentiation in Visual Cortex of Mature Rats. Neuroscience Letters, 342, 196-200. 
https://doi.org/10.1016/S0304-3940(03)00279-9

[108] Shaffery, J.P., Lopez, J. and Roffwarg, H.P. (2012) Brain-Derived Neurotrophic Factor (BDNF) Reverses the Effects of Rapid Eye Movement Sleep Deprivation (REMSD) on Developmentally Regulated, Long-Term Potentiation (LTP) in Visual Cortex Slices. Neuroscience Letters, 513, 84-88.

https://doi.org/10.1016/j.neulet.2012.02.012

[109] Frank, M.G. (2015) Sleep and Synaptic Plasticity in the Developing and Adult Brain. Current Topics in Behavioral Neurosciences, 25, 123-149. https://doi.org/10.1007/7854 2014305

[110] Mirmiran, M., Scholtens, J. and Van De Poll, N.E. (1983) Effects of Experimental Suppression of Active (REM) Sleep during Early Development upon Adult Brain and Behavior in the Rat. Developmental Brain Research, 7, 277-286. https://doi.org/10.1016/0165-3806(83)90184-0

[111] Hobson, J.A. (2005) Sleep Is of the Brain, by the Brain and for the Brain. Nature, 437, 1254-1256. https://doi.org/10.1038/nature04283 